\title{
Management of adhesive intestinal obstruction
}

\author{
A. Niyaf, R. S. Bhandari, K. P. Singh \\ Department of General Surgery, Institute of Medicine, Tribhuvan University Teaching Hospital, Kathmandu, Nepal.
}

Correspondence to: Dr Ali Niyaf, Department of General Surgery, TUTH, Kathmandu, Nepal, Mobile No. +9803792049

Email: ali.niyaf@gmail.com

\begin{abstract}
Introduction: Adhesive intestinal obstruction is an inevitable complication of abdominal surgeries. It has high morbidity with associated poor quality of life and predisposition to repeated hospitalization. Commonest cause of bowel obstruction in developed countries is postoperative adhesions with extrinsic compression of the intestine. Most of them can be managed conservatively.

Methods: A retrospective study of 30 patients admitted with the diagnosis of post operative adhesive partial bowel obstruction was conducted by analyzing their medical records. Demographic data, clinical presentation including duration, previous surgical procedures, treatments received for the condition and successful conservative approach versus requirement of operative intervention were assessed.

Results: The median age was 31 yrs, most in their third decade of life. Male predominance was noted. Pelvic surgeries and gynecological surgeries $(26 \%)$ were found to be the most common cause of adhesive bowel obstruction followed by appendectomy (16\%). More than two third of the patients (76.7\%) developed symptoms within two years of the initial surgery. Successful conservative treatment was noted in 22 patients $(73.3 \%)$ and discharged on fourth day of admission. Eight patients $(26.6 \%)$ underwent surgery. They all underwent adhesiolysis and had good outcome.
\end{abstract}

Conclusions: The time-honored practice of expectant management of adhesive partial bowel obstruction has equally good outcome, as compared to various interventions practiced

Keywords: Adhesive bowel obstruction, conservative management

\section{Introduction}

Small-bowel obstruction is caused by a vast range of pathologies (Table 1). The most common cause in developed countries is postoperative adhesions with extrinsic compression of the intestine. ${ }^{1,2}$ This is followed by malignancy, Crohn's disease and hernias. ${ }^{3}$ Approximately $95 \%$ of adults who undergo abdominal surgery develop bowel obstruction. ${ }^{4}$

Adhesive Intestinal obstruction is an inevitable complication of abdominal surgery with significant morbidity associated with poor quality of life and predispose to repeated hospitalization. Most of them $(73 \%-90 \%)$ can be managed conservatively. ${ }^{5}$ Despite advances in surgery, 15 to $30 \%$ require surgical intervention primarily or due to failure of conservative management. ${ }^{6}$ Because of the nature of the disease recurrence has been estimated to be $30 \%$.

Among surgeries, lower pelvic surgeries are most closely related with postoperative adhesive bowel obstruction. ${ }^{1}$ Surgeries for appendicitis, colorectal pathologies, and gynecologic conditions are noteworthy. Lower abdominal and pelvic surgeries lead to obstruction more often than upper GI surgeries. Small bowel obstruction is mainly classified into complete, partial, simple and strangulated. Although postoperative adhesion is found to be the leading cause of bowel obstruction, numerous other etiologies must not be under emphasized. (Table 1) In case of strangulation, timely emergency surgery is required in order to minimize bowel gangrene and subsequent resection of non viable bowel. 
Intestinal obstruction

Table 1: Common causes of small bowel obstruction

$\begin{array}{lll}\text { Intrinsic causes } & \text { Extrinsic causes } & \text { Intraluminal causes } \\ \text { Neoplasms Inflammatory } & \text { Adhesions } & \text { Intussusception } \\ \text { conditionsAtresia, stenosis } & \text { Hernia } & \text { Meconium/feaces } \\ \text { Congenital Malformation } & \text { Volvulus } & \text { BezoarGallstones } \\ \text { Radiation enteritis } & & \text { Traumatic hematoma }\end{array}$

Strangulation in adhesive obstruction occurs when a loop of distended bowel twists on its mesenteric pedicle which in turn leads to arterial occlusion with subsequent bowel ischemia and necrosis. If not treated in time it may progresses to perforation peritonitis.

Many attempts to prevent formation of postoperative adhesions have been tried. Initial failed procedures such as intra-operative plication of small intestine, insertion of Baker tube "Stitchless Plication" traversing the entire length of small intestine had yielded none but poor results. Chemical agents, such as "Dextran" instillation intra-peritonealy has shown relatively better results. A recent technology which uses Bioresorbable barrier membranes have shown promising results in reducing small bowel obstruction due to adhesions. However large scale research still is needed to prove their efficacy.

As mentioned, treatment of partial bowel obstruction following postoperative adhesions can be conservative sometimes termed "drip and suck" therapy. It cannot be over emphasized that these patients must be investigated and strangulation ruled out. With this said it must be also remembered that these patients must be closely monitored and reassessed for development of strangulation and peritonitis. Optimal length of conservative management is controversial, however until strangulation features are not seen this approach may be tried.

Water soluble oral contrast as Gastrografin also is a respected approach in conservative treatment of adhesive small bowel obstruction. It has been proven to relieve symptoms and minimize hospital stay. Due to its capability for diagnosis as well therapeutic effect, it is gaining popularity. The mechanism is believed to be due to its hypertonic constitution. It causes shift of fluid form edematous intestinal wall into the intestinal lumen. This in turn improves intestinal peristalsis and relieve of obstruction. Surgery is reserved for patients with features of strangulation and these patients must be operated without further delay.

The aim of this study was to evaluate outcome of patients managed with traditional method for partial adhesive intestinal obstruction. Traditionally these patients were conservatively treated with naso-gastric decompression, intravenous fluids supplementation and frequent reassessment for strangulation.

\section{Methods}

A total of 30 patients, who were admitted in the Department of General Surgery, Tribhuvan university teaching hospital with the diagnosis of postoperative incomplete adhesive bowel obstruction from November 2007 to October 2008 were included in this study. These patients' medical records were retrospectively reviewed for their demography, history of previous surgery, the type of surgery, time of presentation from initial symptoms, presenting symptoms. Initial nonoperative management followed by either success or failure of conservative treatment. The rate of conversion to operative treatment and overall outcome were also assessed.

\section{Results}

The median age of the patients who presented with subacute bowel obstruction was 31 years; most of them were in their third decade of life. Male predominance $(56 \%)$ was noted. Pelvic surgeries and gynecological surgeries (26\%) were found to be the most common cause of adhesive bowel obstruction followed by appendectomy (16\%) (Fig. 1).

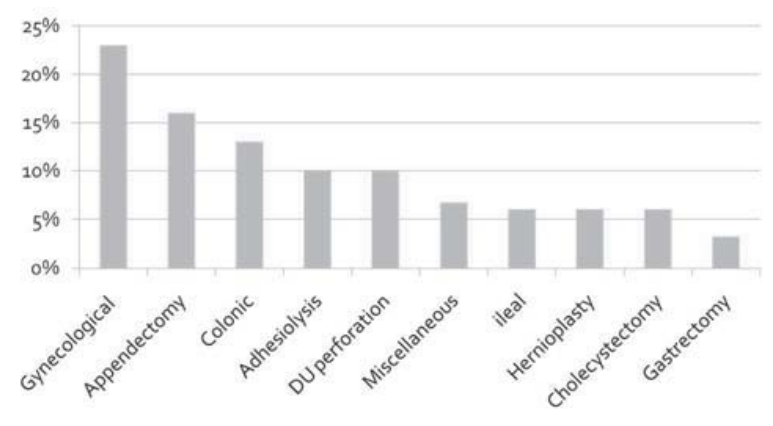

Fig. 1: Incidence of adhesion and surgery

More than one third of the patients $(76.7 \%)$ developed it within two years of the initial surgery. Twenty six patients $(86 \%)$ presented after $24 \mathrm{hrs}$ of initial symptoms. Patients who presented after $48 \mathrm{hrs}$ of symptoms had a noted high 
incidence of operative treatment (Fig.2). Eight patients also gave history of previous similar attack, and were managed conservatively.

Successful conservative treatment was noted in 22 patients $(73.3 \%)$ with discontinuation of naso-gastric decompression within an average period of 2 days followed by discharge on fourth day of admission. Eight patients (26.6\%) underwent surgery due to acute intestinal obstruction. Five of them developed post-operative complications, burst abdomen $(n=2)$ and wound infection $(n=3)$. In all operated cases, the finding was adhesions and bands. They all underwent adhesiolysis and had good outcome.

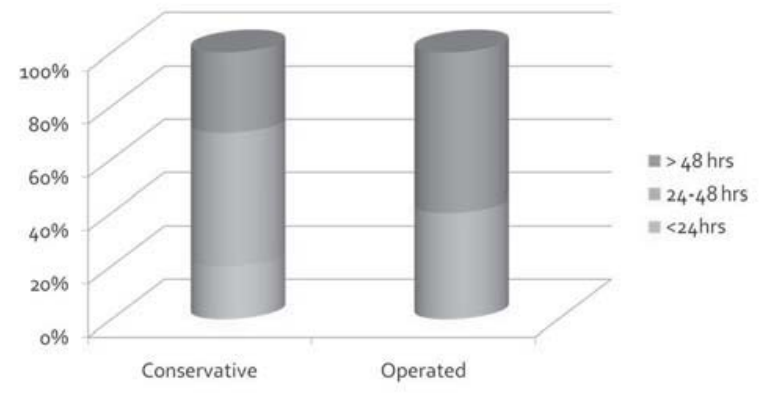

Fig. 2: Correlation of time of presentation and treatment

\section{Discussion}

As all authors agree, safe conservative management needs the pre- requisite of having ruled out any possibility if strangulation of the bowel as the first step of management. This in turn must be followed by frequent vigilant assessment for any progressing symptoms.

In the surgery department at our centre, the management of partial bowel obstruction is based on traditional conservative treatment. Cornerstones are volume resuscitation, bowel decompression with close monitoring for disease progress. This form of management has success rate reported in $75 \%-90 \%$ cases. ${ }^{5}$ Use of adjuvant to conservative treatment has surfaced with the argument to hasten the recovery with decrease in hospital stay and cost. Chen. S, et al investigated the effectiveness of oral laxative, a digestant and a defoaming agent intending to reduce frequency of subsequent surgical intervention and reduce the length of hospital stay. ${ }^{7}$ Chen S. reported more patients in the intervention group than in the control group of having successful treatment without surgery ( $91 \%$ vs. $76 \%$ ).

Biondo $\mathrm{S}$, et al investigated on the therapeutic effect of gastrograffin in adhesive small bowel obstruction and reported of a mean hospital stay of 5.8 days and $77 \%$ successful treatment. ${ }^{8}$ Compared to the expectant management at our center, where the mean hospital stay was 4 days with a successful favorable outcome of $73.3 \%$ only minimal difference is seen with the added advantage of lesser financial burden on the health care system and patient.

\section{Conclusions}

Gynecological surgeries followed by appendectomy are the commonest cause of post-operative adhesive intestinal obstruction. Many trials have been done for hastening the recovery with some promising results. The time-honored expectant management of adhesive partial bowel obstruction practiced in Tribhuvan university teaching hospital has yielded cost effective and equally good outcome.

\section{References}

1. McEntee G, Pender D, Mulvin D, McCullough M, Naeeder S, Farah S, et al. Current spectrum of intestinal obstruction. Br J Surg 1987;74:976-80.

2. Richards WO, Williams LF Jr. Obstruction of the large and small intestine. Surg Clin North Am 1988;68:355-76.

3. Miller G. Etiology of small bowel obstruction. The American Journal of Surgery 2000 ;180(1):33-6

4. Menzies D, Ellis H. Intestinal obstruction from adhesions: How big is the problem? Ann R Coll Surg Engl 1990;72:60-3.

5. Seror D, Feigin E, Szold A, et al. How conservatively can postoperative small bowel obstruction be treated? Am J Surg 1993;165:121-6.

6. Sosa J, Gardner B. Management of patients diagnosed as acute intestinal obstruction secondary to adhesions. Am Surg 1993;59:125-8.

7. Chen S C. Nonsurgical management of partial adhesive small bowel obstruction with oral therapy. CMAJ 2005;173(10).

8. Biondo S. Randomized clinical study of Gastrografin ${ }^{\circledR}$ administration in patients with adhesive small bowel obstruction. BJS 2003;90(5):542-6. 